\title{
RFID Applications in Cyber-Physical System
}

\author{
Nan $\mathrm{Wu}^{1}$ and Xiangdong $\mathrm{Li}^{2}$ \\ ${ }^{1}$ Nanjing University \\ ${ }^{2}$ The City University of New York \\ ${ }^{1}$ China \\ ${ }^{2} U S$
}

\section{Introduction}

A cyber-physical system (CPS) is a system which combines and coordinates the physical system and informatics or computational entities (including computation and communication) into a tight mode. Nowadays we can see the applications of cyber-physical system in the fields of aerospace, automotive, chemical processes, civil infrastructure, energy, healthcare, manufacturing, transportation, entertainment, and consumer appliances.

First, the typical feature of a cyber-physical system is the combination, the CPS is a system deeply combined with computing and physical system. Compared with the so-called embedded system, the percentage of the physical component involved in a CPS is higher than those in an embedded system (shown in Figure 1). In an embedded system, the main focus is on the computational elements, not on the link between the computational and physical elements. Second, unlike a traditional embedded system, usually a CPS is designed as a network of the interaction between the physical input and output, instead of being as a standalone device. The notion is tied to the concepts of robotics and sensor networks. The improvement of the link between computational and physical elements using the advances in science and engineering will boost the use of the cyber-physical systems. Several applications of the use of CPS are "the intervention (e.g., collision avoidance), precision (e.g., robotic surgery and nano-level manufacturing), operation in dangerous or inaccessible environments (e.g., search and rescue, firefighting, and deep-sea exploration), coordination (e.g., air traffic control, war fighting), efficiency (e.g., zero-net energy buildings), and augmentation of human capabilities (e.g., healthcare monitoring and delivery)" [1].

A Radio-frequency Identification (RFID) system is a typical cyber-physical system because of its mainly functional and physical components: (1) The computational element: although a passive RFID tag normally only contains the storage function, but the whole RFID system (mainly in a RFID tag reader) and the post-processing system have the computing and dataprocessing functions; (2) The controlling element: usually a RFID system is under the control of an inner micro- control-unit (MCU); (3) The communication element: in a RFID system, nearly all the information is exchanged via the wave of radio frequency (RF), the data and controlling flows are established via a 2-way RF communication. During the work process, the traditional RFID uses the electronic tags which are placed on the items to track their locations or descriptions. The RFID tags are tiny microchips that can, in some cases, be fabricated smaller than a pinhead or a grain of sand. The chip is attached to a tiny antenna which allows it to communicate and transmit information. Figure 2 is a blown up view of a simple RFID tag [2]. 


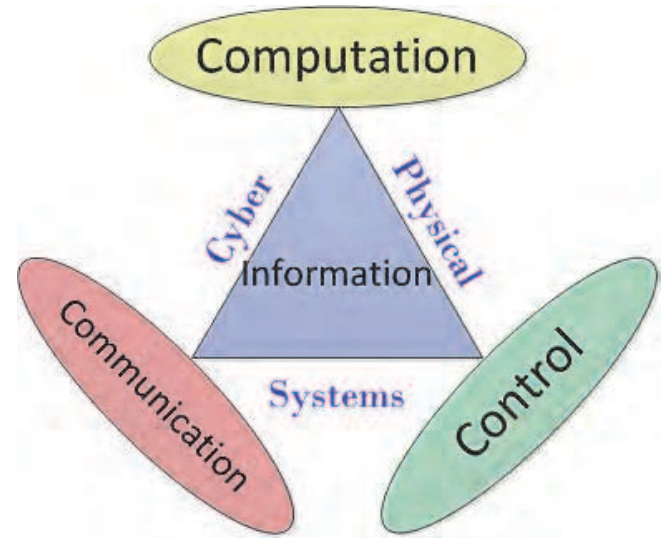

Fig. 1. Three main functional components in a cyber-physical system
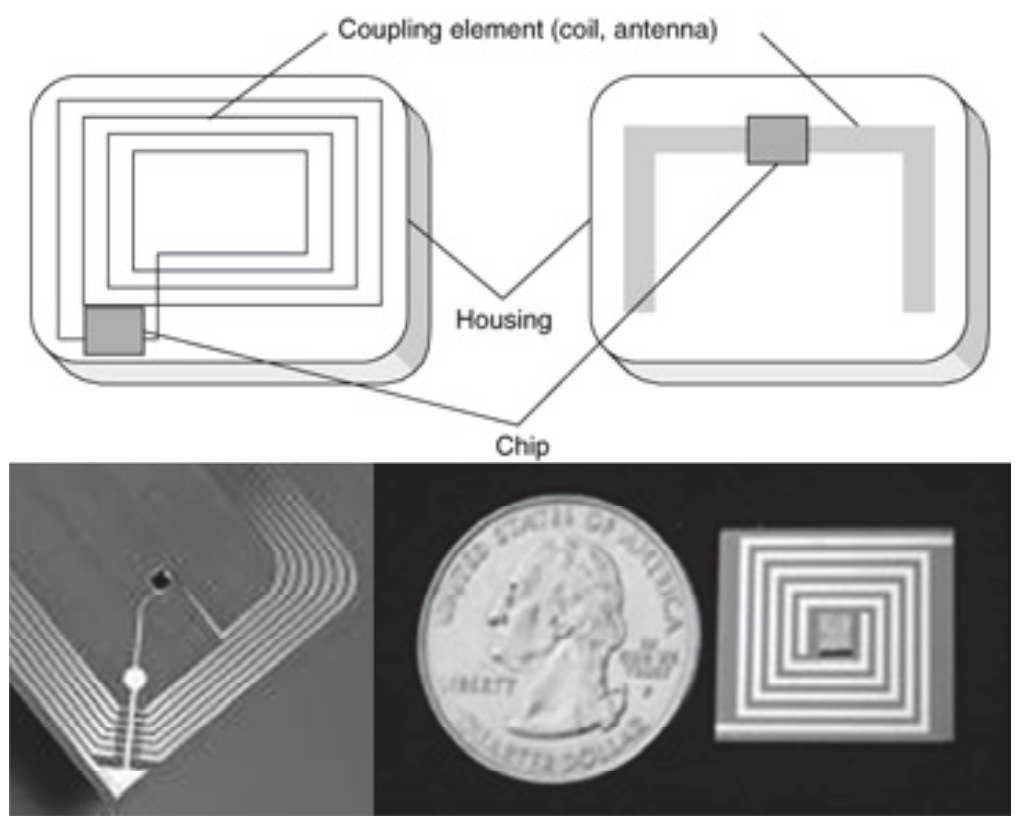

Fig. 2. The structure and outside view of RFID tags

Mostly the regular RFID systems for the civil use are classified into three types - the passive, the semi-passive, and the active RFID. A passive tag is dormant until it is triggered by a signal from a RFID reader. A passive tag does not have a built-in power supply, so it needs the radio frequency energy (electromagnetic wave) from the RFID reader. These tags are particularly popular in use because they can draw the power wirelessly, such that the size and price can be reduced much. Furthermore, these tags can be applied on almost everything because of the wide use of the wireless power supply. A semi-passive tag 
contains a small battery to function an inner timer or random access memory. However, the power supply does not actively communicate with a reader until it is requested. When it is requested, it uses the radio wave power to transmit the information to the reader, which is the same as that of a passive one. An active tag has a more powerful small power source (a battery or other changeable DC source) built-in. Unlike the semi-passive tags, it can actively communicate with the readers without the need of radio wave power.

The most common type of RFID tags used on the market is the passive type and the tags rely on the readers for the energy. A RFID reader usually has a Radio Frequency (RF) module that allows it to transmit and receive messages. It is also manufactured with additional interfaces (e.g. RS 232 or RS485) to allow the connection with the PC's, etc. Figure 3 shows a simple diagram of the communication between a RFID reader and a tag (or transponder). The "application" shown in the diagram is an enterprise network infrastructure.

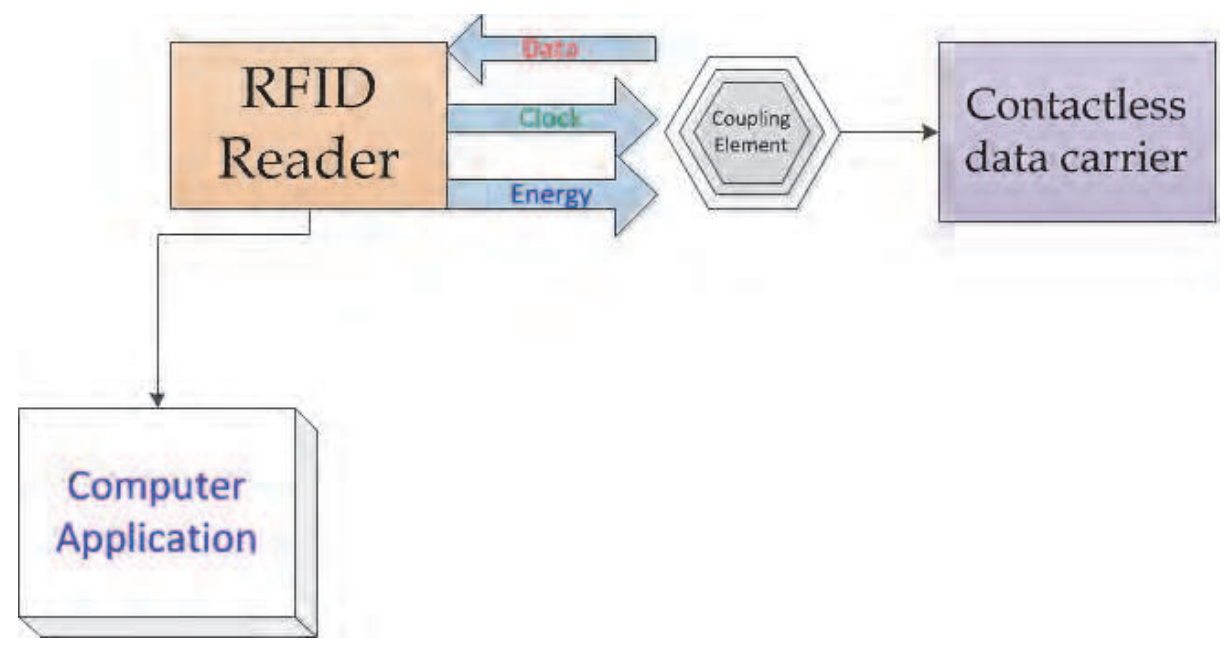

Fig. 3. A Simple passive RFID system diagram

In this chapter, we will study the mixture of a cyber-physical system using the RFID technology. As mentioned above, in a traditional embedded system with a built-in power supplier, using the passive RFID tags is subject to losing the processing ability without the RFID tag readers. To meet the requirements of CPS key application, it is necessary for the RFID tags to contain the batteries and operate the inner MCU and microchips. In the following sections, we will discuss the design on the key applications of the RFID system with the active mode [3].

\section{Active RFID system}

As discussed in the previous section, usually a passive tag holds a unique identification code or a number of 8 bytes in length, along with other small pieces of information. The active and passive tags are different based on the types of information they store. A common passive tag only stores the object identification information, whereas an active tag stores the object description and its transportation history, in addition to the identification information. A real active RFID tag is shown in Figures 4 and 5 [4, 8]. 


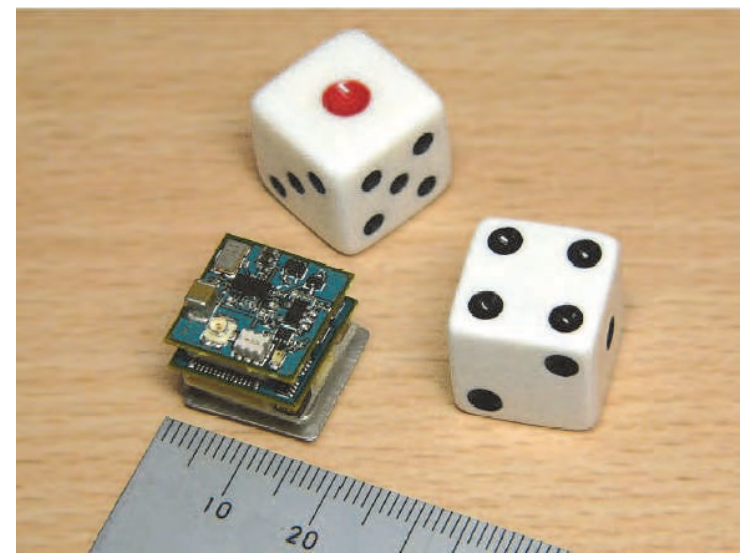

Fig. 4. A compacted active RFID tag

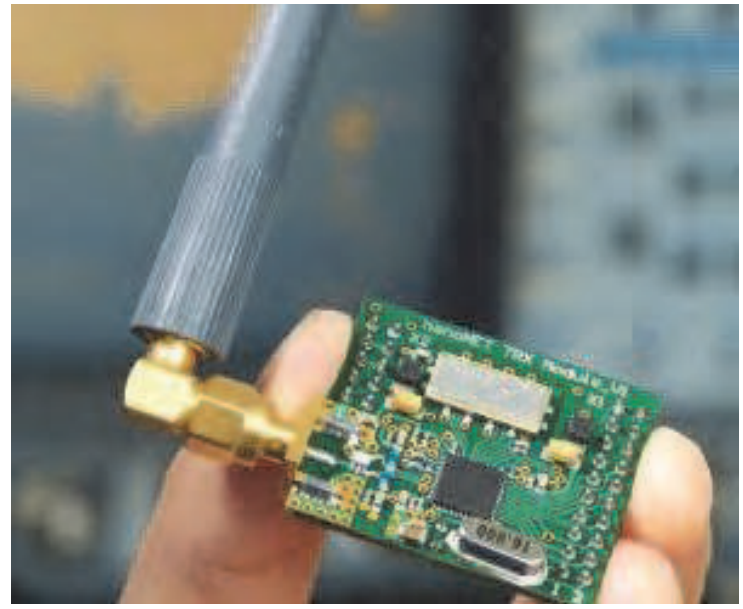

Fig. 5. An active RFID tag with a changeable antenna

To meet the requirements of the key application of cyber-physical system, we should analyze the applicability of a passive RFID system with details. Usually, the microchip in a passive RFID tag is sealed with a plastic cover statically and cannot be altered from its manufacture or configuration. But the information on the tags is able to be rewritten. There are three different core devices which are able to re-write the data into the RFID tags [6]: (1)EEPROMs (electrically erasable programmable read-only memory) are most commonly used among these three. Usually an EEPROM memory capacity ranges from 16 bytes to 8 kilobytes. The disadvantage of using this device for the writing process is the high power consumption. (2)FRAMs (ferromagnetic random access memory)'s reading power consumption is lower than that in the EEPROM. But the manufacturing problems in the past cause an impact on its market acceptance. The FRAMs have a similar limit in the memory capacity. (3)SRAMs (static random access memory) are used especially in the microwave systems and have very high writing cycles. In order to retain the data, it needs an 
uninterrupted power supply, such an auxiliary battery or some other power sources should be equipped for the tags. This obviously limits its usefulness. The SRAMs memory capacity ranges from 256 bytes to 64 kilobytes. From the manufactory experience, a RFID tag can be read and written up to 10 billion times before its performance drops, so the future of this tag is optimistic.

As mentioned in section 1, the application of CPS inclines to "more computation power", the RFID system using passive tags shows several disadvantages when examined with the requirements of CPS applications.

- The processing ability of RFID tags is extremely based on the reader or the connected computers. The tag has a very weak computing ability, so a passive RFID tag is barely as an electrical ID container.

- A passive RFID tag is not able to take any kind of sensor to carry the environment data because of the lack of the driving circuits.

- Even in an active region of a passive RFID reader, the energy supply from the radio coupling of the electromagnetic coil is not sufficient for a more complex computation to function the RFID card's MCU.

- The two-way complex communication is subject to suffering more electromagnetic interference (EMI) during the communications between the card and the reader, plus the radio coupling interaction.

Due to these disadvantages, a passive RFID tag and its reader system cannot meet the requirements of CPS applications. Table 1 is shown in Yamada's research [5]:

\begin{tabular}{|l|l|l|l|}
\hline \multicolumn{1}{|c|}{ Items } & \multicolumn{1}{|c|}{ Passive RFID tag } & Active RFID tag (Con.) & Active RFID tag (new) \\
\hline Comm. Range & $70 \mathrm{~cm} / 3 \mathrm{~m}-7 \mathrm{~m}$ & more than $10 \mathrm{~m}$ & around $10 \mathrm{~m}$ \\
\hline Battery life & (no battery) & around 1 year & around 1 year \\
\hline Security & weak & N/A, or weak & strong \\
\hline Cost & less than \$1 & less than \$10 & around \$10 \\
\hline Application & $\begin{array}{l}\text { distribution/ inventory } \\
\text { control of goods. }\end{array}$ & $\begin{array}{l}\text { tracking person } \\
\text { (restricted area) }\end{array}$ & $\begin{array}{l}\text { tracking person } \\
\text { (no restriction) }\end{array}$ \\
\hline
\end{tabular}

Table 1. Classification of RFID tags

Apparently, an active RFID system can be described highly the likeness of wireless sensor, which has shown to be a successful and mature system. The largest deployment of the active RFID is done by US Department of Defense (DoD), the DoD uses the Savi active tags on each of its over a million shipping containers that travel outside of US.

However, different from a pure wireless sensor system, an active RFID system network is a kind of Ad-hoc network, that is, a heterogeneous network. From the communication protocol point of view, an active RFID reader and its corresponding tags can work with a one-to-many model (and vice versa): one tag can be coupled with many readers (the reader can be defined as a base station in the Ad-hoc model). So when designing the active RFID system protocols, we should consider the difference between the peer-to-peer model and one-to-many model (or many-to-one).

From the network topological structure point of view, a heterogeneous network is wireless based. It is a good carrier for the two-way wireless communication. Here, we define the RFID system used in a CPS system as the followings:

RFID application in CPS $=$ active RFID system + wireless sensor + protocols + network collaborative mechanism 
In the next section we will study a typical RFID application in CPS system.

\section{A typical RFID application in CPS: a case study}

This case study is about the use of an active RFID system which includes a few readers (as the base station) and many active tags (as the sensors) to build an active wireless positioning network, which is a pre-research one of our project [7].

The positioning based services for the geographic information are important in the civil applications, such as the travelling, geographic measurement, harbour operation, driving or logistics; as well as in the military, such as an emergency support or emergency logistics. Today, the global positioning system (GPS) is the most widely used and most well developed positioning system. A GPS receiver uses a high-precision referenced time from a low-orbit satellite to conduct the distance measurement, and it calculates the position by using the geometry methods. The GPS system provides a high positioning accuracy, an excellent timeliness and a strong anti-interference ability. The GPS has many advantages, but with a fatal weakness, that is, its positioning ability and performance are affected distinctly when the receiver is out of the region of the GPS satellite's signal. For example, in an application of the military emergency logistics, when the military vehicle is running in a tunnel or the soldiers are in a thick forest or in a construction, the GPS can not provide the robust positioning service. Especially in a situation such as the need for a rapid response, the loss of GPS performance may cause the possession lost or more casualties. To avoid this problem, the in-door positioning based service is needed for both the military and the civil applications. We study a kind of GPS-independent active positioning system, based on an active RFID system and the TOA (time of arrival) technology and related algorithms [7].

Based on the theory, the distributed node location service uses the referenced base stations (i.e. an active RFID reader, they have the absolute or relative positions of RFID tags) in a distributed network. The node location service is a highly potential core service in the location-based service when applied in a distributed scenario. It shows a great potential, especially when it is used for the positioning in the complicated or blocked indoor/outdoor environment, emergent logistics management, and disaster-relief emergent positioning, etc.

Currently, based on the positioned objects, the distributed node location service's algorithms and the systems can be categorized into a self-node positioning and a target-node positioning. Here we only focus on the self-node positioning. In the positioning technology, a node in the network is recognized as a beacon node or an unknown node based on whether the node is assigned or not assigned with the location information (relative location or absolute location via the GPS or other devices). As the unknown nodes gaining more relative information during the process, therefore, in order to reduce the overall networking loads and the communication cost, the number of the beacon nodes should be limited.

We consider the unknown nodes in the network as the sensors with some special functions (e.g. a function of measuring the distance) and the beacon nodes as the base stations, such that the network with a specific topological structure is a heterogeneous wireless sensor network (WSN). Generally, a well-designed WSN mainly contains the following units:

- Transmission units (including the distance sensors and A/D modules);

- $\quad$ Processing units (including the MCU and embedded software system);

- Communication units (including the radio frequency modules).

We could see clearly that these requirements can be well met based on the active RFID system. In this section we address some key issues on the range based positioning service and study a novel model of the node-location service based on the aforementioned CPS 
model. The theoretical analysis shows that this model can provide a good and stable positioning accuracy and a strong robustness for the scenes where the network topology structure or the node's surrounding environment varies. In addition, it also benefits the reorganizing of the network and the corresponding the active RFID tags' surviving time.

When we combine the node-location service with the proposed model, a new positioning service mode is formulated, that is the wireless sensor network positioning technology. Compared with the traditional wireless positioning technology, it provides new features below:

- Large scale;

- Low hardware resource requirement;

- Non-centralized Ad-hoc network;

- Low energy cost;

- Self-organizing;

- Dynamic topology;

- High positioning accuracy;

- Dynamic positioning supported;

- Communication and positioning;

The accuracy of the range-free positioning service and its convergence rate highly depends on the estimated accuracy on the network's average jumping distance. When the anisotropy or the topological structure of this RFID system's organized WSN becomes complicate, the performance of the algorithm will be significantly weaken. Therefore, compared with the range-free positioning algorithm, the range-based positioning algorithm has such advantages as a better accuracy and a shorter response time. However, on the other hand, it also holds some disadvantages, such as a higher requirement for the hardware and the more power cost. To balance the advantages and the prices, we choose a TOA (Time-of-Arrival)-based distance measurement technology under the WSN as the prototype model.

According to the discussion above, we realize that the in the pursuit of the highly realizable accuracy of the positioning sacrifices the fast response rate and narrow bandwidth with less power cost. An optimization should be reached. We propose a method to simulate and calculate (or optimize) this point by using a statistical model. A lower bound of the positioning accuracy based on the TOA and WSN methods is also discussed in this section.

The algorithm of the TOA costs less hardware resource in the applications, it is helpful to enhance the reliability and robustness of the model. Using the TOA distance estimation, we can apply the trilateration method for the positioning, which is shown in Figure 6.

Considering the mixture of cyber-system and physical system; the aforementioned theory can be designed as algorithms and be coded in MCU of the active RFID tags.

Figure 7 shows the experimental results of the proposed method. The detailed experimental platform is described below.

We physically implemented the specified positioning equipment using the proposed model. The equipment includes an IEEE 802.15.4a chirp spread spectrum (CSS) system, its timedomain and frequency-domain characteristics are shown in the Fig 8. The receiving sensitivity of beacon nodes and unknown nodes is greater than $-97 \mathrm{dBm}$. They work in a duplex mode, cooperating with a gain antenna and a related operating system, and the experiment shows a good signal-to-noise ratio (SNR). The average loaded RF power is only $1 \mathrm{~mW}$, which could significantly ensure the surviving time of the unknown nodes. 


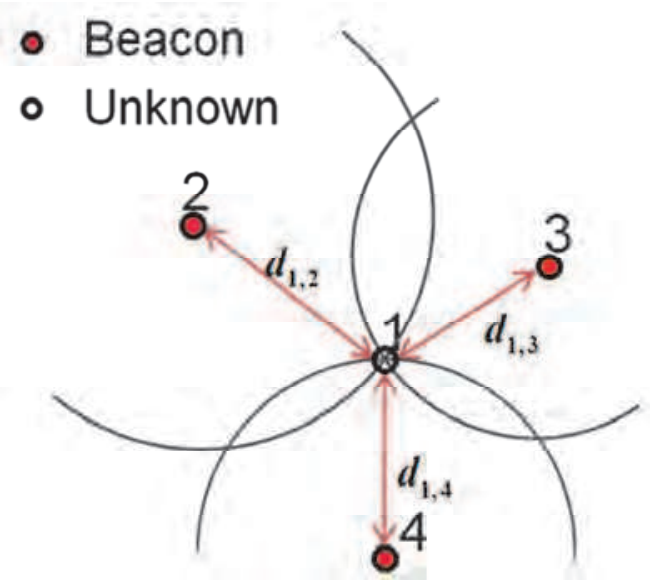

Fig. 6. The estimation of the position of an unknown node by using the trilateration method

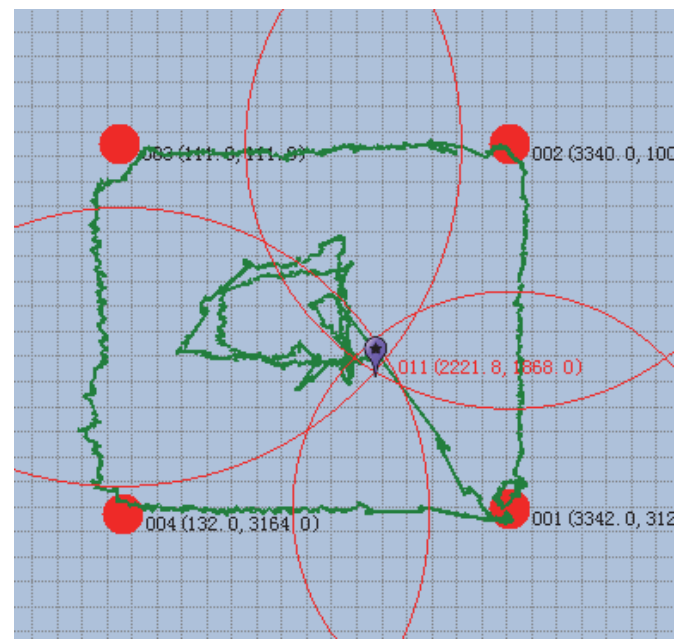

Fig. 7. The experimental results of the bacon-based active position system

The model system has a strong ability of the anti-multi-path-interference and anti-humaninterference. In the modulation, the pulse resolution is adjustable in order to be adapted in different application environments. The networking protocol is uncomplicated and reliable; it can be also added with a 128-bit hardware encryption, which can effectively prevent the interference from outside and the disclosure of the location information.

Based on our prototype of the design, we have made the experimental product. Figure 9 shows the experiment platform which realizes the algorithm and the active RFID system.

We improved this product with a compact size and low-power consumption. The key parameters of the tag are listed in Table 2.

Other product's entities and the related software interface are listed in following Figures 10, 11 and 12. 

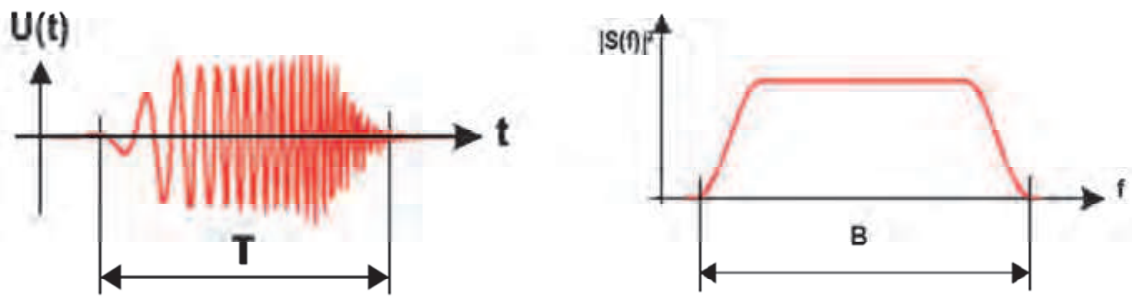

Fig. 8. The time-domain and frequency-domain characteristics of the CSS signal

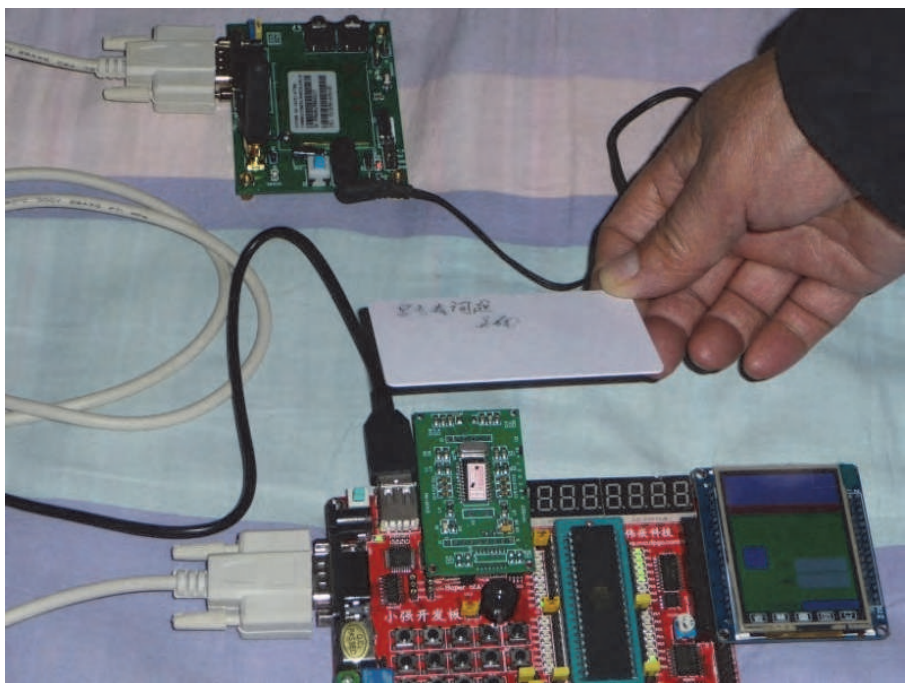

Fig. 9. Experimental platform using RFID reader, tag and wireless transmitting system

\begin{tabular}{|c|c|c|}
\hline Dimensions: & $35 \times 14 \times 2 \mathrm{~mm}$ & \\
\hline Power supply: & $2.7 \mathrm{~V}$ & \\
\hline $\begin{array}{l}\text { Energy } \\
\text { consumption: }\end{array}$ & $0.15 \mathrm{~W}$ & \\
\hline Weight: & $30 \mathrm{~g}$ & $\theta$ \\
\hline Radio frequency: & $2412 \sim 2484 \mathrm{M}$ & \\
\hline Antenna type: & $\begin{array}{l}\text { Built-in (Ceramic or } \\
\text { micro-strip) }\end{array}$ & \\
\hline Installation: & OEM module, SMD & \multirow{3}{*}{$\begin{array}{l}\text { Picture: Chip module and integration } \\
\text { module }\end{array}$} \\
\hline Interface: & TTL, SPI & \\
\hline $\begin{array}{l}\text { Level of } \\
\text { protection: }\end{array}$ & IP65 & \\
\hline
\end{tabular}

Table 2. The compact-sized positioning active RFID tag 

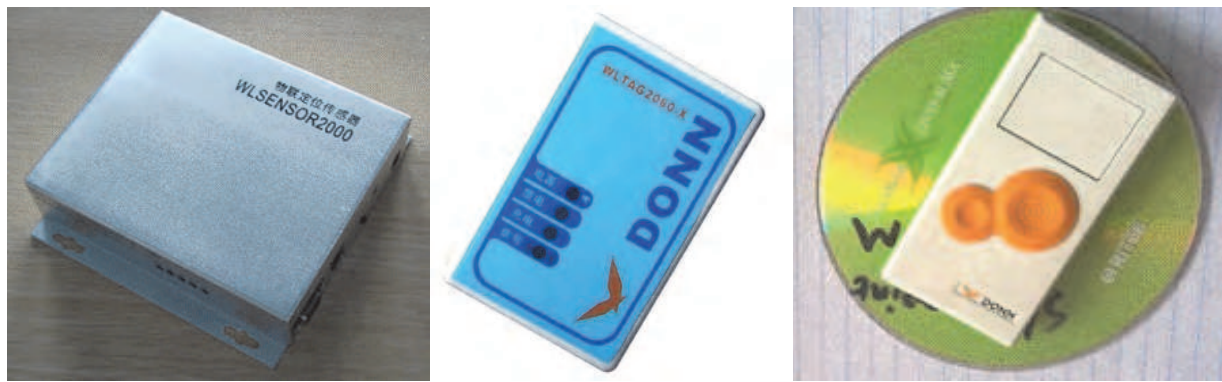

Fig. 10. The indoor positioning sensor and active RFID positioning tag

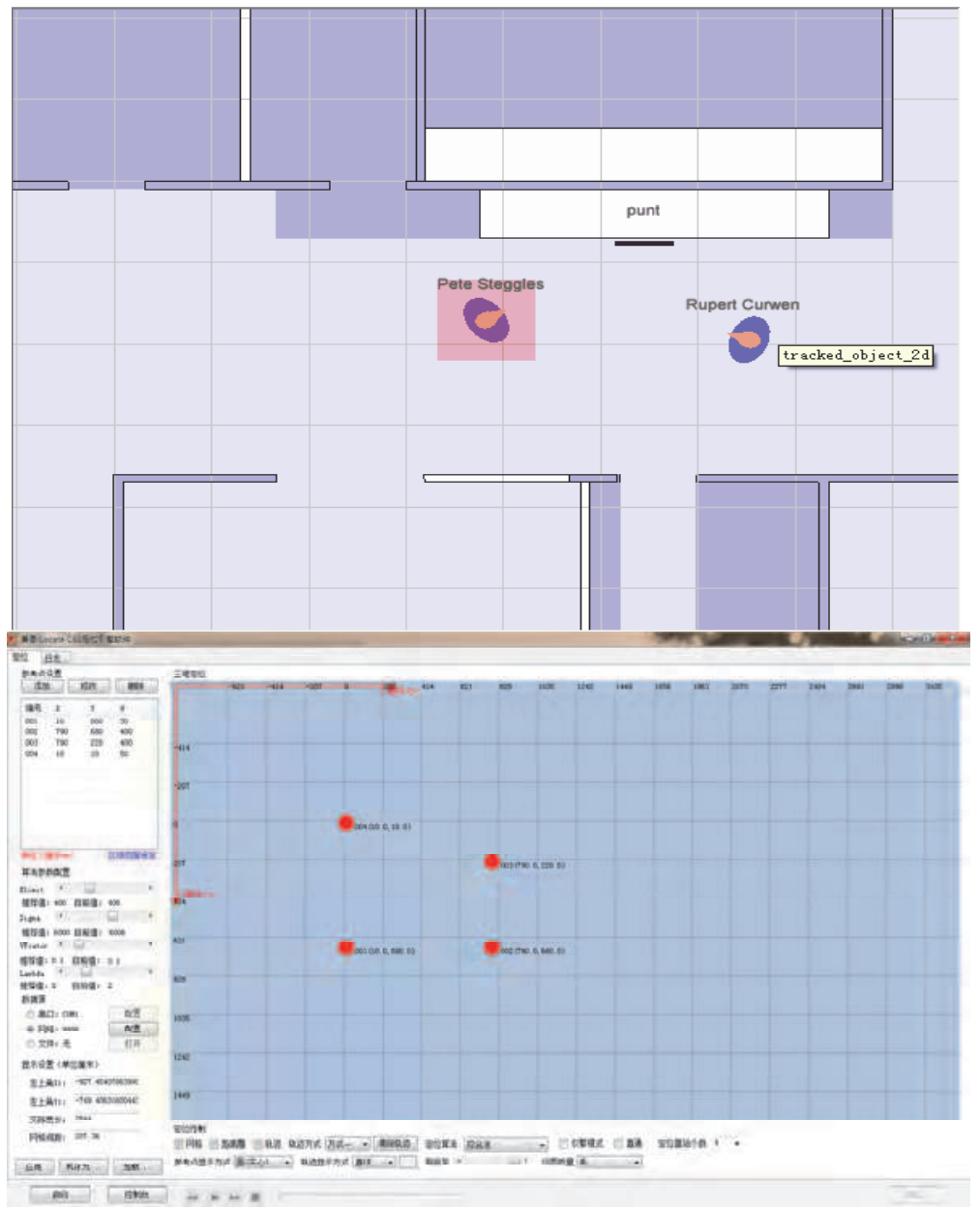

Fig. 11. The related software running on the reader's computer (in 2D mode, red spots indicate the tags' relative position and the blue shadow area is the error space in XY-plane) 


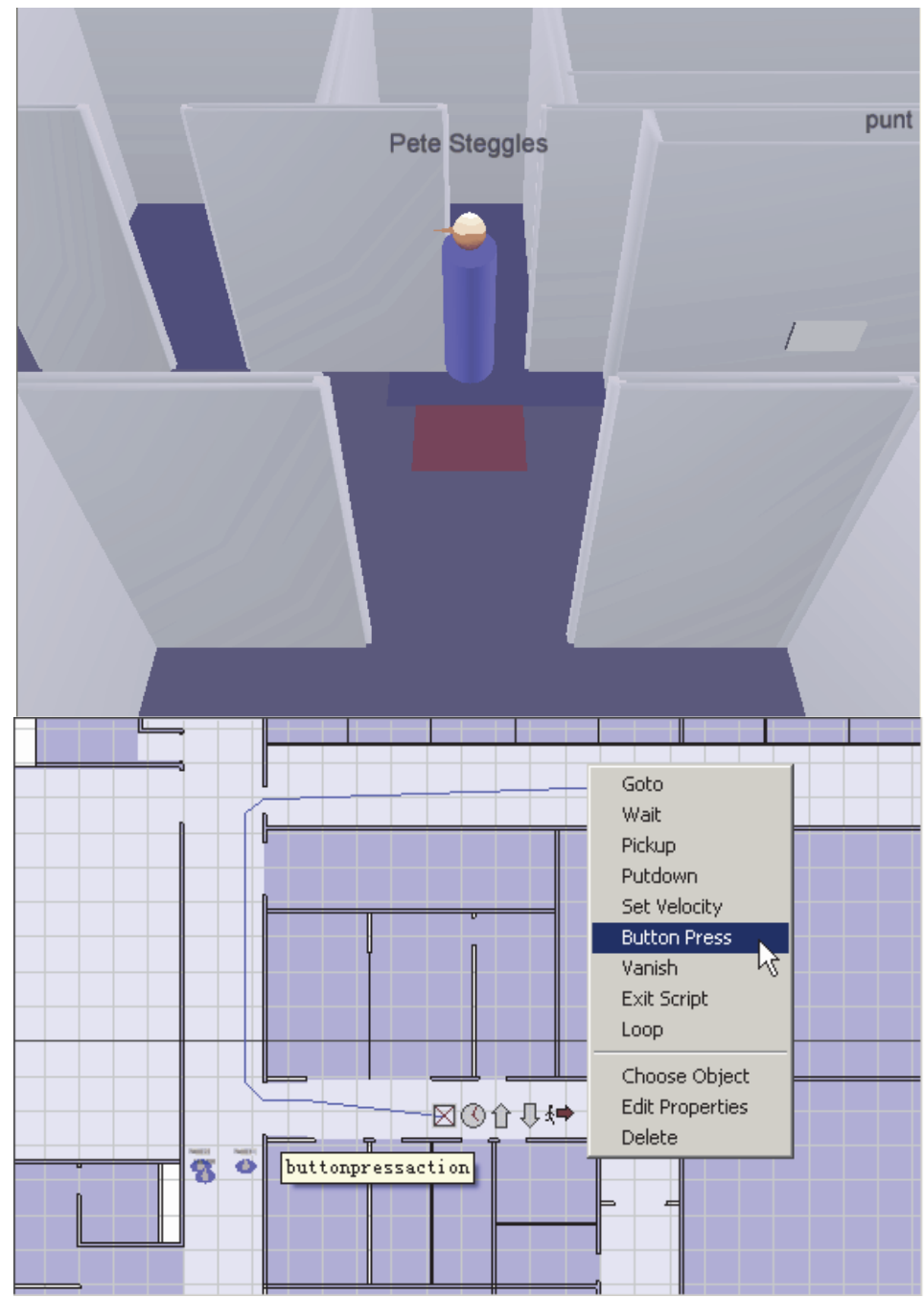

Fig. 12. The related software running on the reader's computer (in $3 \mathrm{D}$ and live-action modes. In the left, the yellow spot means the tags' relative position, the purplish red shadow area is the error space in XY-plane, and the blue cylinder is the height in Z-axial)

\section{Summary}

In conclusion, the active RFID system has shown the gain of a great potential for building a highly-mixed system of information and the physical devices. In this chapter, we compare the RFID system with a traditional wireless sensor network system and discuss the applicability of the type of RFID systems. We propose and study the design idea, methodology, product and experimental results of an active RFID based relative positioning system. 


\section{References}

[1] http://en.wikipedia.org/wiki/Cyber-Physical_Systems

[2] Sztipanovits, J.; Stankovic, J. \& Corman, D. Industry - Academy Collaboration in Cyber Physical System (CPS) Research White Paper V.1 ,Georage Mason University, May 19, 2009

[3] Jalasto, M. Mobile phone applications for radio frequency identification systems, Proc. Reserach Seminar on Telecommunications Bussiness II, Luukkainen S. Eds., April, 2005

[4] http:/ / www.scienceprog.com/how-does-rfid-tag-technology-works/

[5] Yamada, I., Shiotsu, S., Itasaki, A., et al. Secure active RFID tag system, Ubicomp2005 Workshop

[6] Rhodes, J. RFID System Protocols and Standards Overview, Research Report CPET 384 Wide Area Networks, 2007

[7] Lai, X.; Li, J. ; Li, X ; \& Wu, N. A novel model of node location service based on wireless sensor networks and statistical method, Proc. SPIE Wireless Sensing, Localization, and Processing, V, Sohail A. Dianat; Michael D. Zoltowski Eds., 2010

[8] http://tronweb.super-nova.co.jp/tronnews05-4.html 


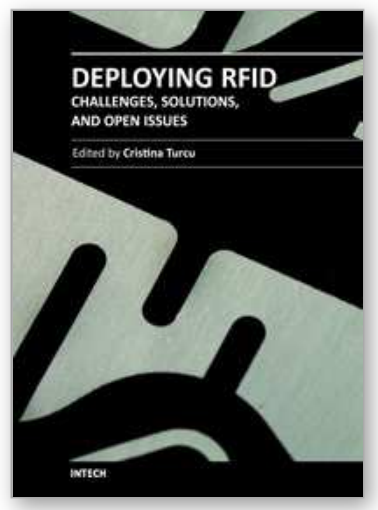

\author{
Deploying RFID - Challenges, Solutions, and Open Issues \\ Edited by Dr. Cristina Turcu
}

ISBN 978-953-307-380-4

Hard cover, 382 pages

Publisher InTech

Published online 17, August, 2011

Published in print edition August, 2011

Radio frequency identification (RFID) is a technology that is rapidly gaining popularity due to its several benefits in a wide area of applications like inventory tracking, supply chain management, automated manufacturing, healthcare, etc. The benefits of implementing RFID technologies can be seen in terms of efficiency (increased speed in production, reduced shrinkage, lower error rates, improved asset tracking etc.) or effectiveness (services that companies provide to the customers). Leading to considerable operational and strategic benefits, RFID technology continues to bring new levels of intelligence and information, strengthening the experience of all participants in this research domain, and serving as a valuable authentication technology. We hope this book will be useful for engineers, researchers and industry personnel, and provide them with some new ideas to address current and future issues they might be facing.

\title{
How to reference
}

In order to correctly reference this scholarly work, feel free to copy and paste the following:

Nan Wu and Xiangdong Li (2011). RFID Applications in Cyber-Physical System, Deploying RFID - Challenges, Solutions, and Open Issues, Dr. Cristina Turcu (Ed.), ISBN: 978-953-307-380-4, InTech, Available from: http://www.intechopen.com/books/deploying-rfid-challenges-solutions-and-open-issues/rfid-applications-incyber-physical-system

\section{INTECH}

open science | open minds

\author{
InTech Europe \\ University Campus STeP Ri \\ Slavka Krautzeka 83/A \\ 51000 Rijeka, Croatia \\ Phone: +385 (51) 770447 \\ Fax: +385 (51) 686166 \\ www.intechopen.com
}

\author{
InTech China \\ Unit 405, Office Block, Hotel Equatorial Shanghai \\ No.65, Yan An Road (West), Shanghai, 200040, China \\ 中国上海市延安西路65号上海国际贵都大饭店办公楼405单元 \\ Phone: +86-21-62489820 \\ Fax: $+86-21-62489821$
}


(C) 2011 The Author(s). Licensee IntechOpen. This chapter is distributed under the terms of the Creative Commons Attribution-NonCommercialShareAlike-3.0 License, which permits use, distribution and reproduction for non-commercial purposes, provided the original is properly cited and derivative works building on this content are distributed under the same license. 